\title{
Flebotomíneos de áreas com notificações de casos autóctones de leishmaniose visceral canina e leishmaniose tegumentar americana em Angra dos Reis, Rio de Janeiro, Brasil
}

\author{
Marcos Barbosa de Souzaㄹ, Raimundo Wilson de Carvalho², Roberto Nei Martins Machado ${ }^{1}$ \\ \& Eduardo Dias Wermelinger ${ }^{1}$
}

${ }^{1}$ Laboratório de Vetores, Departamento de Ciências Biológicas, Rua Leopoldo Bulhões, 1480, Manguinhos-Rio de Janeiro 21041-210 ENSP/FIOCRUZ.mabaza@ensp.fiocruz.br; rmachado@ensp.fiocruz.br; edw@ensp.fiocruz.br

${ }^{2}$ Laboratório de Ixodides, Pavilhão Mourisco, Avenida Brasil, 4365, Manguinhos-Rio de Janeiro, Instituto Oswaldo Cruz - FIOCRUZ. raicar@ioc.fiocruz.br

\begin{abstract}
Phlebotomine fauna in areas of autochthonous cases of canine visceral leishmaniasis and American cutaneous leishmaniasis in Angra dos Reis, Rio de Janeiro, Brazil. The city of Angra dos Reis presents human cases of American cutaneous leishmaniasis since 1945. Phlebotomine surveys in 1978 had disclosed the presence of Nyssomyia intermedia and the first notification of Lutzomyia longipalpis in Rio de Janeiro, Ilha Grande, Angra dos Reis. In August of 2002 the first canine case of visceral leishmaniasis in the Ilha Grande, Angra dos Reis was notified. Surveys of phlebotomines in the peridomiciliary areas, in the period of November, 2002, through May, 2003, in four localities of Angra dos Reis, resulted in 12,554 specimens belonging to nine species: Brumptomyia sp. Nyssomyia intermedia, Migonemyia migonei, Micropygomyia schreiberi, Pintomyia fischeri, Psychodopygus davisi, Psychodopygus ayrosai, Evandromyia tupinambay, Psathyromyia pelloni. Nyssomyia intermedia was predominant in all localities, followed by M. migonei. The main vector of the AVL, L. longipalpis, was not detected in the localities, including their surroundings, where an infected dog inhabited.
\end{abstract}

KEYWORDS. American visceral leishmaniasis; Phlebotomine sandfly.

RESUMO. Flebótomos de áreas com notificações de casos autóctones de leishmaniose visceral canina e leishmaniose tegumentar americana em Angra dos Reis, Rio de Janeiro, Brasil. O município de Angra dos Reis apresenta casos humanos de leishmaniose tegumentar americana desde 1945. Inquéritos flebotomínicos realizados em 1978 revelaram a presença de Nyssomyia intermedia e a primeira notificação de Lutzomyia longipalpis no Rio de Janeiro, Ilha Grande, Angra dos Reis. Em agosto de 2002 foi notificado o primeiro caso canino de leishmaniose visceral na Ilha Grande, Angra dos Reis. Inquéritos flebotomínicos realizados nos peridomicílios, no período de novembro de 2002 a maio de 2003 , em quatro localidades de Angra dos Reis, resultaram em 12.554 flebotomíneos e a presença de nove espécies: Brumptomyia sp.; Nyssomyia intermedia, Migonemyia migonei, Micropygomyia schreiberi, Pintomyia fischeri, Psychodopygus davisi, Psychodopygus ayrosai, Evandromyia tupinambay, Psathyromyia pelloni. foi Nyssomyia intermedia, predominante em todas as localidades, seguida por M.migonei. O principal vetor da LVA, Lutzomyia longipalpis, não foi detectado nas localidades, incluindo áreas do entorno, onde um cão infectado residia.

PALAVRAS-CHAVE. Flebotomíneos; leishmaniose visceral americana.

A Leishmaniose Tegumentar Americana (LTA) é endêmica no estado do Rio de Janeiro desde o inicio do século passado. Com relação à ocorrência de casos humanos de LTA na Ilha Grande, há notificações de casos desde 1945, onde, um imigrante adquiriu a doença no povoado de Freguesia de Santana, a 17 quilômetros da Praia Vermelha, sendo posteriormente registrado em 1965, novo caso autóctone na mesma localidade (Araújo Filho 1978). Após estudos epidemiológicos o autor diagnosticou 33 casos autóctones desta protozoose entre 1975 e 1976.

A Leishmaniose Visceral Americana (LVA) foi registrada pela primeira vez no Estado do Rio de Janeiro em 1978, no município do Rio de Janeiro (Marzochi et al. 1985).
Em 1983 foram notificados 35 casos autóctones em áreas peri-urbanas do Rio de Janeiro, situadas nas vertentes continentais norte, noroeste, oeste e sudeste do Maciço da Pedra Branca ( Marzochi et al. 1985).

O principal vetor da LVA no Brasil, Lutzomyia longipalpis, foi em seguida apontado por evidências epidemiológicas, como vetor desta protozoose no município do Rio de Janeiro (Souza et al. 1981). Esta espécie já havia sido coletada no município de Mangaratiba e Angra dos Reis, anteriormente a ocorrência dos primeiros casos humanos e caninos de LVA (Lutz \& Neiva 1912; Costa Lima 1932 e Araújo Filho 1980).

O Estado do Rio de Janeiro é assinalado como área de transmissão esporádica de LVA, entretanto, esta doença vem 
se dispersando para diferentes áreas do município do Rio de Janeiro (Souza et al. 2003), Angra dos Reis (Caso canino e Humano 2002), Mangaratiba (1984 e 2001) e Itaguaí (2006) (dados não publicados).

Tendo em vista a ocorrência de casos de Leishmanioses em Angra dos Reis, principalmente a notificação do primeiro caso canino de LVA em Ilha Grande, forte pólo turístico internacional, aliado a escassez de estudos relacionados a vetores de leishmanioses, foram realizados inquéritos flebotomínicos na região da Praia do Abraão, Ilha Grande e no continente do município de Angra dos Reis.

Angra dos Reis situa-se na Região Sudeste, Rio de Janeiro, Brasil, na Baía da Ilha Grande, Latitude S: entre $23^{\circ} 05^{`}$ e $23^{\circ} 14^{\prime}$ - Longitude W: entre $44^{\circ} 05^{\prime}$ e $44^{\circ} 23^{\prime}$. A Ilha Grande apresenta uma área de $193 \mathrm{~km}$ quadrados, perímetro do contorno igual a $130 \mathrm{~km}$, acidentado com 34 pontas, sete enseadas e 106 praias. Sendo uma ilha oceânica, tem influência marinha acentuada no clima local, o qual é tropical, quente e úmido, com temperatura variando entre $15^{\circ} \mathrm{C}-30^{\circ} \mathrm{C}$. A temperatura média anual alcança $22,5^{\circ} \mathrm{C}$, sendo fevereiro o mês mais quente, $25,7^{\circ} \mathrm{C}$ e julho, o mais frio, $19,6^{\circ} \mathrm{C}$. A precipitação média anual é de $2.242 \mathrm{~mm}$, sendo janeiro o mês mais chuvoso (293 mm) e julho, o menos chuvoso $(87 \mathrm{~mm})$. A topografia é montanhosa com vários picos, sendo os de maior altitude o da Pedra d'agua com $1031 \mathrm{~m}$ e o do Papagaio com $982 \mathrm{~m}$. Predominam na área o relevo com dissecações extremamente fortes e muito fortes, constituídas de cristais de topos aguçados, morros, pontões e escarpas.

Conforme os dados obtidos do Departamento de Vigilância Epidemiológica e Fiscalização Sanitária de Angra dos Reis, foram notificados dois casos caninos de LTA e um de LVA na Vila do Abraão, Ilha Grande e casos humanos de LTA no continente.

Na parte continental do município de Angra dos Reis, foi selecionada uma residência onde residia um cão com LVA no Bairro de Verolme. Outras localidades com notificações de casos humanos de LTA, também foram selecionadas: Camorim, Brachuy e Caputera. Na Ilha Grande as coletas se concentraram na Vila do Abraão, principalmente no Camping onde o cão infectado permanecia nos períodos de temporada (dezembro a fevereiro) e na periferia deste local. As atividades de coleta foram divididas entre duas equipes com quatro profissionais cada uma responsável pelas coletas de flebótomos nas áreas do continente e a segunda pelas coletas da Vila do Abraão, Ilha Grande.

As coletas foram mensais, em três noites consecutivas, nos anexos dos peridomicílios e nos domicílios, utilizando captura manual (com capturador de castro), de novembro de 2002 a maio de 2003. O horário de capturas foi estabelecido entre $18 \mathrm{~h}$ e $23 \mathrm{~h}$, ou seja, 5 horas captura/técnico/local, perfazendo um total de 35 horas de capturas por área. Os flebótomos coletados foram fixados em álcool $70 \%$ e transportados para o laboratório, onde foram clarificados em potassa $10 \%$ ( 2 horas), ácido acético 10\% (15 minutos), lavagem em água destilada (três passagens de 5 minutos) e lactofenol $(24 \mathrm{~h})$. Posteriormente, foram montados e identificados com base nas descrições e chaves de identificação Galati (2003).

Foram capturados 12.554 flebotomíneos (Tab. I), pertencentes a nove espécies: Brumptomyia sp., Nyssomyia intermedia (Lutz \& Neiva, 1912), Psychodopygus davisi (Root, 1934), P. ayrozai (Barretto \& Coutinho, 1940), Migonemyia migonei (França, 1920), Micropygomyia schreiberi (Martins, Falcão \& Silva, 1975), Pintomyia fischeri (Pinto, 1926), Evandromyia tupynambai (Mangabeira, 1942) e Psathyromyia pelloni (Sherlock \& Alencar, 1959).

Apenas um exemplar de Brumptomyia sp. foi capturado na localidade da Vila do Abraão, em um galinheiro próximo a mata Atlântica. Em Verolme, no continente, segundo domicílio cujo cão infectado costumava freqüentar não foi notificada a presença de flebótomos (Tab. II). Nas demais áreas do continente foram coletados: 3.159 flebótomos em Bracuy, 3.139 em Camorim e 746 em Caputera, totalizando 7.044 exemplares, cujas espécies mais freqüentes foram N.intermedia, M.migonei, M.schreiberi e as demais espécies com densidades reduzidas (P. ayrosai, P. davisi, P.fischeri ). Na Vila do Abraão, Ilha Grande, o número de exemplares foi similar aos obtidos no continente, totalizando 5.510 flebótomos, com as seguintes espécies: Brumptomyia sp., N.intermedia, M.migonei, P.pelloni, E.tupynambay e M.schreiberi (Tab. I).

A predominância de machos sobre fêmeas foi acentuada,

Tabela I. Número e percentuais de flebótomos coletados no município de Angra dos Reis, Rio de Janeiro.

\begin{tabular}{|c|c|c|c|c|c|c|c|c|c|c|c|c|}
\hline \multirow[b]{2}{*}{ Espécies } & \multicolumn{4}{|c|}{ I. Grande (Abraão) } & \multicolumn{4}{|c|}{ Angra dos Reis(*) } & \multicolumn{4}{|c|}{ Total } \\
\hline & $\sigma^{x}$ & $\%$ & 우 & $\%$ & $0^{x}$ & $\%$ & 우 & $\%$ & $\sigma^{x}$ & $\%$ & 우 & $\%$ \\
\hline Nyssomyia intermedia & 3617 & 92.7 & 1284 & 79.9 & 4127 & 92.2 & 2337 & 91 & 7744 & 92.41 & 3621 & 86.8 \\
\hline Migonemyia migonei & 239 & 6.1 & 297 & 18.5 & 316 & 7.1 & 199 & 7.8 & 555 & 6.62 & 496 & 11.9 \\
\hline Pintomyia fischeri & 0 & 0 & 0 & 0 & 2 & 0.04 & 3 & 0.1 & 2 & 0.03 & 3 & 0.07 \\
\hline Psychodopygus davisi & 0 & 0 & 0 & 0 & 0 & 0 & 2 & 0.07 & 0 & 0 & 2 & 0.04 \\
\hline Evandromyia tupinambay & 1 & 0.02 & 4 & 0.25 & 0 & 0 & 0 & 0 & 1 & 0.01 & 4 & 0.09 \\
\hline Psychodopygus ayrosai & 0 & 0 & 0 & 0 & 0 & 0 & 14 & 0.53 & 0 & 0 & 14 & 0.3 \\
\hline Psathyromyia pelloni & 3 & 0.06 & 0 & 0 & 0 & 0 & 0 & 0 & 3 & 0.04 & 0 & 0 \\
\hline Micropygomyia schreiberi & 43 & 1.1 & 21 & 1.31 & 31 & 0.69 & 13 & 0.5 & 74 & 0.88 & 34 & 0.8 \\
\hline Brumptomyia sp. & 1 & 0.02 & 0 & 0 & 0 & 0 & 0 & 0 & 1 & 0.01 & 0 & 0 \\
\hline Total & 3904 & 100 & 1606 & 99.96 & 4476 & 100.03 & 2568 & 100 & 8380 & 100 & 4174 & 100 \\
\hline
\end{tabular}

Angra dos Reis $(*)$ - Localidades de Bracuy - Caputera e Camorim 
Tabela II. Total geral de flebótomos coletados no Continente de Angra dos Reis, Rio de Janeiro. Período de Novembro de 2002 a Setembro de 2003.

\begin{tabular}{|c|c|c|c|c|c|c|c|c|c|c|}
\hline \multirow[t]{2}{*}{ Espécies } & \multicolumn{2}{|c|}{ Bracuy } & \multicolumn{2}{|c|}{ Caputera } & \multicolumn{2}{|c|}{ Camorim } & \multicolumn{2}{|c|}{ Verolme } & \multicolumn{2}{|c|}{ Total } \\
\hline & $\mathrm{N}^{\mathrm{o}}$ & $\%$ & $\mathrm{~N}^{\mathrm{o}}$ & $\%$ & $\mathrm{~N}^{\mathrm{o}}$ & $\%$ & $\mathrm{~N}^{\mathrm{o}}$ & $\%$ & $\mathrm{~N}^{\mathrm{o}}$ & $\%$ \\
\hline Nyssomyia intermedia & 2886 & 91.4 & 598 & 80.2 & 2980 & 95 & 0 & 0 & 6464 & 91.8 \\
\hline Migonemyia migonei & 235 & 7.4 & 135 & 18.1 & 145 & 4.6 & 0 & 0 & 515 & 7.3 \\
\hline Psychodopygus ayrozai & 13 & 0.4 & 0 & 0 & 1 & 0.02 & 0 & 0 & 14 & 0.2 \\
\hline Pintomyia fischeri & 2 & 0.06 & 0 & 0 & 3 & 0.08 & 0 & 0 & 5 & 0.07 \\
\hline Psychodopigus davisi & 2 & 0.06 & 0 & 0 & 0 & 0 & 0 & 0 & 2 & 0.03 \\
\hline Micropygomyia schreiberi & 21 & 0.7 & 13 & 1.7 & 10 & 0.3 & 0 & 0 & 44 & 0.6 \\
\hline Total & 3159 & 100 & 746 & 100 & 3139 & 100 & 0 & 0 & 7044 & 100 \\
\hline
\end{tabular}

8.380 machos e 4.174 fêmeas entretanto, na Vila do Abraão ocorreu ligeira predominância das fêmeas (297) sobre os machos (239) de Lutzomyia migonei.

Com relação às frequiências mensais foram obtidos no continente de Angra dos Reis, maiores densidades nos meses de novembro (2012), dezembro de 2002 (1956) e maio de 2003 (1484). Na Vila do Abraão as freqüências obtidas foram: novembro (1574), dezembro de 2002 (1836) e maio de 2003 (1174). Apesar da ocorrência de caso canino autóctone de LVA, não foi detectada a presença da L.longipalpis na Praia do Abraão.

O município de Angra dos Reis apresentou entre 1991 e 2003 a incidência de 341 casos humanos autóctones de LTA superado apenas pelos municípios do Rio de Janeiro e de Paraty, com 940 e 714 casos respectivamente (Registros das Secretarias Municipais de Saúde e Sistema de Informação de Agravos e Notificação - SINAN).

A baixa diversidade de espécies neste trabalho ocorreu em função das coletas terem sido realizadas em ambientes urbanos na Ilha Grande, bem como das áreas trabalhadas no continente. As interferências ecológicas do ambiente antrópico são demonstradas claramente pelas adaptações e pelo alto número de espécimes de N.intermedia e Migonemyia migonei, predominantes nas áreas endêmicas de LTA no Estado do Rio de Janeiro (Lima et al. 1988; Souza et al. 2003; Carvalho et al.1995).

A captura de espécies com comportamento silvestre, como Psychodopygus ayrozai, Evandromyia tupinambay e Psychodopygus davisi, ocorreu pela influência dos ecótopos florestais que estão muito próximos das áreas sob ação antrópica, embora com reduzido número de exemplares. Devese ressaltar que as espécies P.ayrosai e P.davisi apresentam importância na epidemiologia da transmissão da Leishmania (Viannia) naiffi, o primeiro na região da Amazônia (Rangel \& Lainson 2003) e a segunda no Estado de Roraima (Gil et al. 2003).

Foi relatada a ocorrência de um caso humano autóctone e inédito de leishmaniose cutânea difusa (Leishmania amazonensis) no município vizinho de Paraty, onde até o momento não há evidencias de ocorrência de Bichoromomyia flaviscutelata (Agencia Fiocruz de Notícias 2007). Em trabalhos realizados em área sob a influencia da Serra do Mar, em Itaguai, Rio de Janeiro, Aguiar et al.(1996), não notificaram a presença das espécies $P$. ayrosai e $P$. davisi, mesmo trabalhando em ambiente silvestre, entretanto, estes mesmos autores notificaram a presença da $L$. longipalpis em altitudes superiores a $300 \mathrm{~m}$. Na Praia do Abraão, a altitude é inferior a $50 \mathrm{~m}$ de altitude. Souza et al. (1981) constataram que a $L$. longipalpis apresentava maior frequiência em altitudes maiores a $100 \mathrm{~m}$ em áreas com ocorrência de caso humano autóctone de LVA na zona Oeste do município do Rio de Janeiro. Da Costa et al. (2004) realizaram inquéritos flebotomínicos em diferentes localidades do município de Paraty e não constataram a presença de $P$. ayrosai e $P$. davisi nos ambientes peridomiciliares da região.

Lutzomyia longipalpis não foi notificada na Praia do Abraão e nas demais áreas trabalhadas no continente. O cão infectado percorria toda a Ilha Grande, sendo possível que tenha adquirido a LVC em outra região, pois Araújo Filho (1980) registrou L. longipalpis na Praia Vermelha.

Quanto à transmissão da LTA no município de Angra dos Reis sugere-se que esteja sendo veiculada pelas espécies N.intermedia e M.migonei, ambas notificadas recentemente com infecção natural por Leishmania (Viannia) braziliensis no município do Rio de Janeiro (Pitta Pereira et al. 2005).

\section{REFERÊNCIAS}

Alencar, J. E. 1978. Leishmaniose visceral no Brasil. Revista de Medicina da Universidade Federal do Ceará 17-18: 129-48. Araújo-Filho, N. A.; I. A. Shrelock \& J. R. Coura. 1980. Leishmaniose Tegumentar Americana na Ilha Grande, Rio de Janeiro. VI. Observações sobre a freqüência horária e variação mensal dos transmissores. Revista da Sociedade Brasileira de Medicina Tropical 14: 185-195.

Aguiar, G. M.; W. M. Medeiros; T. S. De Marco; S. T. Santos, de \& S. Gambardella. 1996. Ecologia dos flebotomíneos da Serra do Mar, Itaguaí, Estado do Rio de Janeiro, Brasil. I - A fauna flebotomínica e prevalência pelo local e tipo de captura (Diptera, Psychodidae, Phlebotominae). Cadernos de Saúde Pública 12: 195-206.

Agencia Fiocruz de Notícia. [On line]. Disponivel na Internet. URL:http://www.fiocruz.br/ccs/cgi/cgilua.exe/sys/start.htm. Acesso em 14 de maio de 2007.

Carvalho, R. W.; N. M. Serra-Freire \& M. B. Souza. 1995. Fauna de flebótomos da Ilha do Araújo, município de Paraty-RJ. 1 Diversidades e aspectos do comportamento. Parasitología Al Día 19: 104-112.

Costa Lima, A. 1932. Sobre os Phlebótomos Americanos (Diptera, Psychodidae). Memórias do Instituto Oswaldo Cruz 26: 15-69. 
Costa, C. M.; F. F. B. Moutinho \& S. F. Bruno. 2004. A experiência do município de Paraty (Rio de Janeiro, Brasil) na prevenção e controle da leishmaniose tegumentar americana. Parasitología Latinoamericana 59: 110-114.

Forattini, O. P. 1973. Entomologia Médica, São Paulo, Edgard Blücher, Universidade de São Paulo, vol 4, 658 pp.

Galati, E. A. B. 2003. Classificação de Phlebotominae. In: Rangel E.F. \& R. Lainson. Flebotomíneos do Brasil. Rio de Janeiro: Ed. Fiocruz. p. 23-51.

Gil, L. H. S; A. S. Basano; A. A. Souza; M. G. S. Silva; I. Barata \& E. Ishikawa. 2003. Recent observations on the sand fly (Diptera: Psychodidae) fauna of the State of Rondônia, Western Amazônia, Brazil: the importance of Psychdopygus davisi as a vector of zoonotic cutaneous leishmaniasis. Memórias do Instituto Oswaldo Cruz 98: 751-5.

Lima, L. C. R.; M. C. A. Marzochi; P. C. Sabroza \& M. A. Souza. 1988. Observações sobre a Leishmaniose Tegumentar cinco anos após profilaxia. Revista de Saúde Pública 22: 73-7.

Lutz, A. \& A. Neiva. 1912. Contribuição para o conhecimento das espécies do gênero Phlebotomus existentes no Brasil. Memórias do Instituto Oswaldo Cruz 4: 84-95.

Marzochi, M. C. A.; P. C. Sabroza; L. M. Toledo; K. B. Marzochi; N. C. Tramontano \& F. B. Rangel Filho. 1985. Leishmaniose Visceral na cidade do Rio de Janeiro- Brasil. Cadernos de Saúde Pública 1: $5-17$.
Pita-Pereira, D.; C. R. Alves; M. B. Souza; R. P. Brazil; Á. L. Bertho; A. F. Barbosa \& C. C. Britto. 2005. Identification of naturally infected Lutzomyia intermedia and Lutzomyia migonei with Leishmania (Viannia) braziliensis in Rio de Janeiro (Brazil) revealed by a PCR multiplex non-isotopic hybridisation assay. Transactions of the Royal Society of Tropical Medicine and Hygiene 99: 905913.

Rangel, E. F. \& R. Lainson. 2003. Ecologia das leishmanioses: transmissores de leishmaniose tegumentar americana. In: Rangel, E. F. \& R. Lainson. (org.). Flebotomíneos do Brasil. Rio de Janeiro: Editora Fiocruz, 291-310.

Souza, M. A. de; P. C. Sabroza, M. C. A. Marzochi; S. G. Coutinho \& W. J. S. Souza, 1981. Leishmaniose visceral no Rio de Janeiro. 1 Flebotomíneos da área de procedência de caso humano autóctone. Memórias do Instituto Oswaldo Cruz 76: 161-168.

Souza, M. B.; J. R. Pujol-Luz; M. C. A. Marzochi; R. W. Carvalho; C. S. Ponte \& A. M. Meira. 2000. Estudo da fauna flebotomínica em área de Leishmanioses Tegumentar Americana e Visceral, zona periurbana do bairro de Jacarepaguá, Rio de Janeiro. Revista Entomologia y Vetores 17: 365-375.

Souza, M. B.; M. C. A. Marzochi; R. W. Carvalho; P. C. Ribeiro; C. S. Ponte; J. M. Caetano \& A. M. Meira. 2003. Ausência da Lutzomyia longipalpis em algumas áreas de ocorrência de leishmaniose visceral no Município do Rio de Janeiro. Caderno de Saúde Pública 19 $1881-1885$

Recebido em 10/09/2007; aceito em 19/09/2008 